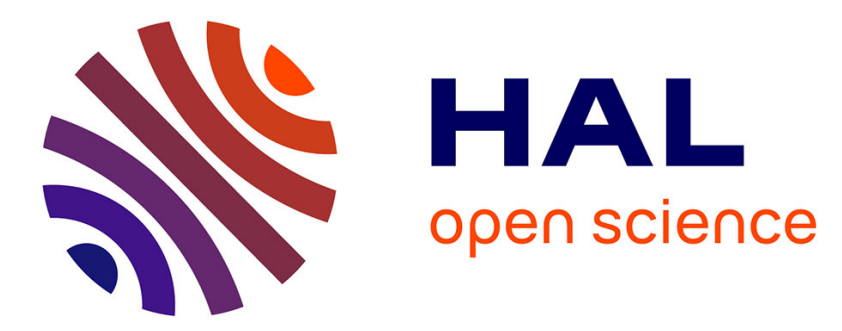

\title{
Contextuality in multipartite pseudo-telepathy graph games
}

\author{
Anurag Anshu, Peter Høyer, Mehdi Mhalla, Simon Perdrix
}

\section{To cite this version:}

Anurag Anshu, Peter Høyer, Mehdi Mhalla, Simon Perdrix. Contextuality in multipartite pseudotelepathy graph games. Journal of Computer and System Sciences, 2020, 107, pp.156-165. 10.1016/j.jcss.2019.06.005 . hal-02400051

\section{HAL Id: hal-02400051 \\ https://hal.science/hal-02400051}

Submitted on 21 Dec 2021

HAL is a multi-disciplinary open access archive for the deposit and dissemination of scientific research documents, whether they are published or not. The documents may come from teaching and research institutions in France or abroad, or from public or private research centers.
L'archive ouverte pluridisciplinaire HAL, est destinée au dépôt et à la diffusion de documents scientifiques de niveau recherche, publiés ou non, émanant des établissements d'enseignement et de recherche français ou étrangers, des laboratoires publics ou privés.

\section{다)(1) $(5$}

Distributed under a Creative Commons Attribution - NonCommerciall 4.0 International 


\title{
Contextuality in Multipartite Pseudo-Telepathy Graph Games
}

\author{
Anurag Anshu ${ }^{\mathrm{a}}$, Peter Høyer ${ }^{\mathrm{b}}$, Mehdi Mhallac ${ }^{\mathrm{c}}$, Simon Perdrix ${ }^{\mathrm{d}}$ \\ ${ }^{a}$ Centre for Quantum Technologies, National University of Singapore \\ ${ }^{b}$ University of Calgary, Canada \\ ${ }^{c}$ Univ. Grenoble Alpes, CNRS, Grenoble INP, LIG, F-38000 Grenoble France \\ ${ }^{d}$ CNRS, LORIA, Université de Lorraine, Inria Carte, Nancy, France
}

\begin{abstract}
Analyzing pseudo-telepathy graph games, we propose a way to build contextuality scenarios exhibiting the quantum supremacy using graph states. We consider the combinatorial structures generating equivalent scenarios. We introduce a new tool called multipartiteness width to investigate which scenarios are hard to decompose and show that there exist graphs generating scenarios with a linear multipartiteness width.
\end{abstract}

Keywords: Quantum Information, Graph States, Contextuality, Multipartite Entanglement

\section{Introduction}

Contextuality is an active area of research that describes models of correlations and interpretations, and links to some fundamental questions about the natural world. It also provides a framework where one can utilize the understanding of quantum mechanics (and quantum information) in order to better analyze, understand, and interpret macroscopic phenomena [10, 34, 24, 21, 49].

The theoretical and experimental study of quantum world has proven that a scenario involving many parties (each having access to a local information) can contain correlations that do not possess any classical interpretation that relies on decomposition of these correlations using local functions. Frameworks for contextuality provide a tool to describe the combinatorial structures present in these correlations.

Contextuality has been well studied in literature, see for instance [14, 48], and a large family of them $[7,2,45,6,3]$ are based on a model introduced by 
Abramsky and Brandenburger [1] which uses sheaf theory to naturally relate issues surrounding the consistency of interpretation to the pre-sheaf structure obtained by a distribution functor on the sheaf of events. The authors introduce three levels of contextuality: (i) Probabilistic contextuality, which corresponds to the possibility of simulating locally and classically a probability distribution over outcomes for each allowed context of measurements. It extends the celebrated Bell's theorem [12] which shows that quantum probabilities are inconsistent with the predictions of any local realistic theory; (ii) Logical contextuality or possibilistic contextuality, which generalizes the kind of contextuality present in Hardy's construction [32] and considers only the support of a probability distribution; (iii) Strong contextuality, which generalizes the kind of contextuality present in local measurements of the GHZ state [29] and relies on the existence of a global assignment consistent with the support.

More recently Acín, Fritz, Leverrier, and Belén Sainz [8] have presented contextuality scenarios defined as hypergraphs, in which vertices correspond to outcomes and hyperedges are called measurements. A general interpretation model is an assignment of non negative reals to the vertices that can be interpreted as a probability distribution for any hyperedge (weights of the vertices of each hyperedge sum to 1 ). Each hypergraph $H$ admits a set $\mathcal{C}(H)$ (resp. $\mathcal{Q}(H), \mathcal{G}(H)$ ) of classical (resp. quantum, general probabilistic) models with $\mathcal{C}(H) \subseteq \mathcal{Q}(H) \subseteq \mathcal{G}(H)$.

They have shown that the Foulis Randall product of hypergraphs [25] allows one to describe the set of no-signaling models in product scenarios $\mathcal{G}\left(H_{1} \otimes H_{2}\right)$. They have also investigated the multipartite case, showing that the different products for composition of local interpretation models produce models that are observationally equivalent.

Non locality is a particular case of contextuality, exhibited for example in the pseudo-telepathy games [13]. These are games that can be won by non-communicating players that share quantum resources, but cannot be won classically without communication.

A family of pseudo-telepathy games based on graph states have been introduced in [9]. The pseudo-telepathy game associated with a graph $G$ of order $n$ (on $n$ vertices), is a collaborative $n$-player game where each player receives a binary input (question) and is asked to provide, without communication, a binary output (answer). Some global pairs of (answers - questions) are forbidden and correspond to losing positions. We associate a hypergraph to each pseudotelepathy game, with its vertices labelled by pairs (answers - questions) 
representing a contextuality scenario. In order to quantify its multipartiteness, we define the multipartiteness width: a scenario built from a game on $n$ parties has a multipartiteness width less than $k$ if it has an interpretation (assignment of real positive numbers to the vertices) that can be obtained interpretations of contextual scenarios on less than $\mathrm{k}$ parties as resources.

It has been shown in [17] that even though GHZ type scenarios are maximally non local (strongly contextual), they can be won with 2 partite nonlocal boxes. So the multipartiteness width is different from the usual measures of contextuality $[28,4]$. However, it has potential application for producing device independent witnesses for entanglement depth [37].

In section 2, we define the graph pseudo-telepathy games, investigate in detail the quantum strategy and link them to contextuality scenarios. The quantum strategy consists in sharing a particular quantum state called graph state [33]. Graph states have multiple applications in quantum information processing, e.g. secret sharing [40, 27, 39], interactive proofs [15, 38, 44], and measurement-based quantum computing [46, 22, 23, 18, 43, 42]. We show in section 3 that provided that the players share multipartite randomness, it is enough to perfectly win the associated pseudo-telepathy game, in order to simulate the associated quantum probability distribution. In section 4, we prove that graphs obtained by a combinatorial graph transformation called pivoting correspond to equivalent games. Finally, we prove that there exist graphs for which the multipartiteness width is linear in the number of players, improving upon the previous logarithmic bound given in [9].

Note that even though the rules of these graph games appear non-trivial, they naturally correspond to the correlations present in outcomes of a quantum process that performs $X$ and $Z$ measurements on a graph state. Thus, they might be easy to produce experimentally. Furthermore even if the space of events is quite large, the scenarios have the advantage of possessing concise descriptions, quite similar to the separating scenarios using Johnson graphs in [26]. Requiring such large structures to achieve possibilistic contextuality for quantum scenarios seems to be unavoidable. Indeed, it has been shown that multiparty XOR type inequalities involving two-body correlation functions cannot achieve pseudo-telepathy [30]. 


\section{Pseudo-telepathy graph games, multipartiteness and contextu- ality scenarios}

Graph notations. We consider finite simple undirected graphs. Let $G=$ $(V, E)$ be a graph. For any vertex $u \in V, N_{G}(u)=\{v \in V \mid(u, v) \in E\}$ is the neighborhood of $u$. For any $D \subseteq V$, the odd neighborhood of $D$ is the set of all vertices which are oddly connected to $D$ in $G$ : $\operatorname{Odd}(D)=\{v \in V$ : $|D \cap N(v)|=1 \bmod 2\} . \operatorname{Even}(D)=V \backslash \operatorname{Odd}(D)$ is the even neighborhood of $D$, and $\operatorname{loc}(D)=D \cup \operatorname{Odd}(D)$ is the local set of $D$ which consists of the vertices in $D$ and those oddly connected to $D$. See Figure 1.

For any $D \subseteq V, G[D]=(D, E \cap D \times D)$ is the subgraph induced by $D$, and $|G[D]|$ its size, i.e. the number of edges of $G[D]$. Note that Odd can be realized as a linear map (where we consider subsets as binary vectors), which implies that for any two subset of vertices $A, B, \operatorname{Odd}(A \oplus B)=\operatorname{Odd}(A) \oplus \operatorname{Odd}(B)$ where $\oplus$ denotes the symmetric difference.

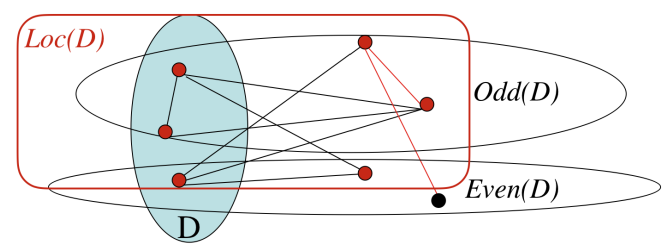

Figure 1: Even and odd neighborhoods.

We introduce the notion of involvement:

Definition 1 (Involvement). Given a graph $G=(V, E)$, a set $D \subseteq V$ of vertices is involved in a binary labelling $x \in\{0,1\}^{V}$ of the vertices if $D \subseteq \operatorname{supp}(x) \subseteq \operatorname{Even}(D)$, where $\operatorname{supp}(x)=\left\{u \in V \mid x_{u}=1\right\}$.

In other words, $D$ is involved in the binary labelling $x$, if all the vertices in $D$ are labelled with 1 and all the vertices in $\operatorname{Odd}(D)$ are labelled with 0 . Notice that when $G[D]$ is not a union of Eulerian graphs ${ }^{1}$, there is no binary labelling in which $D$ is involved. On the other hand, if $G[D]$ is a union of Eulerian graphs, there are $2^{|\operatorname{Even}(D)|-|D|}$ binary labellings in which $D$ is involved.

\footnotetext{
${ }^{1}$ The following three properties are equivalent: (i) $D \subseteq \operatorname{Even}(D)$; (ii) every vertex of $G[D]$ has an even degree; (iii) $G[D]$ is a union of Eulerian graphs. Notice that $D \subseteq \operatorname{Even}(D)$ does not imply that $G[D]$ is Eulerian as it may not be connected.
} 
Collaborative games. A multipartite collaborative game $\mathcal{G}$ for a set $V$ of players is a scenario characterised by a set $\mathcal{L} \subseteq\{0,1\}^{V} \times\{0,1\}^{V}$ of losing pairs: each player $u$ is asked a binary question $x_{u}$ and has to produce a binary answer $a_{u}$. The collaborative game is won by the players if for a given question $x \in\{0,1\}^{V}$ they produce an answer $a \in\{0,1\}^{V}$ such that the pair formed by $a$ and $x$, denoted $(a \mid x)$, is not a losing pair, i.e. $(a \mid x) \notin \mathcal{L}$.

A game is pseudo-telepathic if classical players using classical resources cannot perfectly win the game (unless they cheat by exchanging messages after receiving the questions) whereas using entangled states as quantum resources the players can perfectly win the game, giving the impression to a quantum non believer that they are telepathic (as the only classical explanation to a perfect winning strategy is that they are communicating).

An $n$-player strategy here is viewed as a protocol without communication where each player has access to some kind of resources (shared randomness, quantum states, $k$-partite non local boxes) that they access locally in a sequence using a finite number of rounds before producing their output. We use the term classical to refer to the case where the players have access only to shared randomness.

Example 1: The losing set associated with the Mermin parity game [41] is $\mathcal{L}_{\text {Mermin }}=\left\{(a \mid x): \sum x_{i}=0 \bmod 2\right.$ and $\left.\sum a_{i}+\left(\sum x_{i}\right) / 2=1 \bmod 2\right\}$. Notice that the losing set admits the following simpler description: $\mathcal{L}_{\text {Mermin }}=$ $\{(a \mid x): 2|a|=|x|+2 \bmod 4\}$, where $|x|=|\operatorname{supp}(x)|$ is the Hamming weight of $x$.

Collaborative graph games $\mathrm{MCG}(\mathbf{G})$ : A multipartite collaborative game MCG $(G)$ associated with a graph $G=(V, E)$, where $V$ is a set of players, is the collaborative game where the set of losing pairs is $\mathcal{L}_{G}:=\{(a \mid x): \exists D$ involved in $x$ s.t. $\left.\sum_{u \in \operatorname{loc}(D)} a_{u}=|G[D]|+1 \bmod 2\right\}$. In other words, the collaborative game is won by the players if for a given question $x \in\{0,1\}^{V}$ they produce an answer $a \in\{0,1\}^{V}$ such that for any non-empty $D$ involved in $x$,

$$
\sum_{u \in \operatorname{loc}(D)} a_{u}=|G[D]| \bmod 2
$$

Example 2: Consider $\operatorname{MCG}\left(K_{n}\right)$ the collaborative game associated with the complete graph $K_{n}$ of order $n$. When a question $x$ contains an even number of $1 \mathrm{~s}$ the players trivially win since there is no non-empty subset of vertices involved in such a question. When $x$ has an odd number of $1 \mathrm{~s}$, the 
set of players (vertices) involved in this question is $D=\operatorname{supp}(x)$. In this case, all the players are either in $D$ or $\operatorname{Odd}(D)$ thus the sum of all the answers has to be equal to $|G[D]|=\frac{|D|(|D|-1)}{2}=\frac{|D|-1}{2} \bmod 2$. Thus for the complete graph $K_{n}, \mathcal{L}_{K_{n}}=\left\{(a \mid x):|x|=1 \bmod 2\right.$ and $\left.|a|=\frac{|x|-1}{2}+1 \bmod 2\right\}=$ $\{(a \mid x): 2|a|=|x|+1 \bmod 4\}$. Note that for this particular graph, the constraints are global in the sense that the sum of the answers of all the players is used for all the questions. Notice also that the set of losing pairs $\mathcal{L}_{K_{n}}=\{(a \mid x): 2|a|=|x|+1 \bmod 4\}$ is similar to the one of the Mermin parity game, $\mathcal{L}_{\text {Mermin }}=\{(a \mid x): 2|a|=|x|+2 \bmod 4\}$. In section 4 , we actually show the two games simulate each other.

Quantum strategy (Qstrat): In the following we show that for any graph $G$, the corresponding multipartite collaborative game can be won by the players if they share a particular quantum state. More precisely the state they share is the so-called graph state $|G\rangle=\frac{1}{\sqrt{2^{|V|}}} \sum_{y \in\{0,1\}^{V}}(-1)^{|G[\operatorname{supp}(y)]|}|y\rangle$, and they apply the following strategy: every player $u$ measures his qubit according to $X$ if $x_{u}=1$ or according to $Z$ if $x_{u}=0$. Every player answers the outcome $a_{u} \in\{0,1\}$ of this measurement.

This quantum strategy QStrat, not only produces correct answers, but provides all the good answers uniformly:

Lemma 2. Given a graph $G=(V, E)$ and question $x \in\{0,1\}^{V}$, the probability $p(a \mid x)$ to observe the outcome $a \in\{0,1\}^{V}$ when each qubit $u$ of a graph state $|G\rangle$ is measured according to $Z$ if $x_{u}=0$ or according to $X$ if $x_{u}=1$ satisfies:

$$
p(a \mid x)= \begin{cases}0 & \text { if }(a \mid x) \in \mathcal{L} \\ \frac{\mid\{D \text { involved in } x\} \mid}{2^{|V|}} & \text { otherwise. }\end{cases}
$$

Proof: According to the Born rule, the probability to get the answer $a \in$ $\{0,1\}^{V}$ to a given question $x \in\{0,1\}^{V}$ is:

$$
\begin{aligned}
p(a \mid x) & =\left\langle G\left|\left(\bigotimes_{v \in V \backslash \operatorname{supp}(x)} \frac{I+(-1)^{a_{v}} Z_{v}}{2}\right) \otimes\left(\bigotimes_{u \in \operatorname{supp}(x)} \frac{I+(-1)^{a_{u}} X_{u}}{2}\right)\right| G\right\rangle \\
& =\frac{1}{2^{n}} \sum_{D \subseteq V}(-1)^{\sum_{u \in D} a_{u}}\left\langle G\left|Z_{D \backslash \operatorname{supp}(x)} X_{D \cap \operatorname{supp}(x)}\right| G\right\rangle
\end{aligned}
$$

The basic property which makes this strategy work is that for any $u \in V$, $X_{u}|G\rangle=Z_{N(u)}|G\rangle$. As a consequence, since $X$ and $Z$ anti-commute and 
$X^{2}=Z^{2}=I$, for any $D \subseteq V, X_{D}|G\rangle=(-1)^{|G[D]|} Z_{\operatorname{Odd}(D)}|G\rangle$. Thus, $p(a \mid x)=\frac{1}{2^{n}} \sum_{D \subseteq V}(-1)^{|G[D \cap \operatorname{supp}(x)]|+\sum_{u \in D} a_{u}}\left\langle G\left|Z_{(\operatorname{Odd}(D \cap \operatorname{supp}(x))) \oplus(D \cap \backslash \operatorname{supp}(x))}\right| G\right\rangle$

Where $\oplus$ denotes the symmetric difference. Since $\left\langle G\left|Z_{C}\right| G\right\rangle=\left\{\begin{array}{ll}1 & \text { if } C=\emptyset \\ 0 & \text { otherwise }\end{array}\right.$,

$$
\begin{aligned}
p(a \mid x) & =\frac{1}{2^{n}} \sum_{D \subseteq V, D \backslash \operatorname{supp}(x)=\operatorname{Odd}(D \cap \operatorname{supp}(x))}(-1)^{|G[D \cap \operatorname{supp}(x)]|+\sum_{u \in D} a_{u}} \\
& =\frac{1}{2^{n}} \sum_{D_{1} \subseteq \operatorname{supp}(x)} \sum_{D_{0} \subseteq V \backslash \operatorname{supp}(x), D_{0}=\operatorname{Odd}\left(D_{1}\right)}(-1)^{\left|G\left[D_{1}\right]\right|+\sum_{u \in D_{0} \cup D_{1}} a_{u}} \\
& =\frac{1}{2^{n}} \sum_{D_{1} \subseteq \operatorname{supp}(x), \operatorname{Odd}\left(D_{1}\right) \cap \operatorname{supp}(x)=\emptyset}(-1)^{\left|G\left[D_{1}\right]\right|+\sum_{u \in \operatorname{loc}\left(D_{1}\right)} a_{u}} \\
& =\frac{1}{2^{n}} \sum_{D_{1} \text { involved in } x}(-1)^{\left|G\left[D_{1}\right]\right|+\sum_{u \in \operatorname{loc}\left(D_{1}\right)} a_{u}}=\frac{\left|R_{0}^{(x, a)}\right|-\left|R_{1}^{(x, a)}\right|}{2^{n}}
\end{aligned}
$$

where $R_{d}^{(x, a)}=\left\{D\right.$ involved in $\left.x:|G[D]|+\sum_{u \in \operatorname{loc}(D)} a_{u}=d \bmod 2\right\}$. If $(a \mid x) \notin \mathcal{L}$, then $R_{1}^{(x, a)}=\emptyset$, so $p(a \mid x)=\frac{\mid\{D \text { involved in } x\} \mid}{2^{n}}>0$ since $\emptyset$ is involved in $x$. Otherwise, there exists $D^{\prime} \in R_{1}^{(x, a)}$. Notice that $R_{0}^{(x, a)}$ is a vector space $\left(\forall D_{1}, D_{2} \in R_{0}^{(x, a)}, D_{1} \oplus D_{2} \in R_{0}^{(x, a)}\right)$ and $R_{1}^{(x, a)}$ an affine space $R_{1}^{(x, a)}=$ $\left\{D^{\prime} \oplus D \mid D \in R_{0}^{(x, a)}\right\}$. Thus $\left|R_{0}^{(x, a)}\right|=\left|R_{1}^{(x, a)}\right|$ which implies $p(a \mid x)=0$.

The probability distribution produced by QStrat depends on the number of sets $D$ involved in a given question $x$. Notice that a set $D \subseteq \operatorname{supp}(x)$ is involved in $x$ if and only if $D \in \operatorname{Ker}\left(L_{x}\right)$, where $L_{x}$ linearly ${ }^{2}$ maps $A \subseteq \operatorname{supp}(x)$ to $\operatorname{Odd}(A) \cap \operatorname{supp}(x)$. Thus $\mid\{D$ involved in $x\} \mid=2^{|x|-r k_{G}(x)}$, where $r k_{G}(x)=$ $\left.\log _{2}\left(\mid\left\{L_{x}(A): A \subseteq \operatorname{supp}(x)\right)\right\} \mid\right)$ is the rank of $L_{x}=A \mapsto \operatorname{Odd}(A) \cap \operatorname{supp}(x)$.

\section{Contextuality scenario.}

With any multipartite collaborative game $\mathbf{M C G}(G)$, we associate a hypergraph which describes a contextuality scenario in the sense of the hypergraph approach to contextuality[8]. The vertices are the pairs $(a \mid x)$ and each hyperedge corresponds, roughly speaking, to a constraint.

\footnotetext{
${ }^{2} L_{x}$ is linear for the symmetric difference: $L_{x}\left(D_{1} \oplus D_{2}\right)=L_{x}\left(D_{1}\right) \oplus L_{x}\left(D_{2}\right)$
} 
The hyperedges can be decomposed into two subsets : those $\left(H_{\mathrm{Nsig}_{V}}\right)$ which guarantee no-signaling and those $\left(H_{G}\right)$, depending on the graph $G$, which avoid the losing pairs:

- $H_{\mathrm{Nsig}_{V}}$ is the hypergraph representing the no-signaling polytope. It corresponds [8] to the Bell scenario $B_{|V|, 2,2}$ where $|V|$ parties have access to 2 local measurements each, each of which has 2 possible outcomes (see Figure 2), which is obtained as a product ${ }^{3}$ of the elementary scenario $B_{1,2,2}$.

- The hypergraph $H_{G}$ defined on the same vertex set, corresponds to the game constraints: for each question ${ }^{4} x \in\{0,1\}^{V}$ we associate an hyperedge $e_{x}$ containing all the answers which make the players win on $x$ i.e., $e_{x}=\left\{(a \mid x) \in\{0,1\}^{V} \times\{0,1\}^{V},(a \mid x) \notin \mathcal{L}\right\}$.

Given a graph $G=(V, E), \operatorname{MCG}(G)$ is a pseudo-telepathy game if it admits a quantum model $\left(\mathcal{Q}\left(H_{G} \cup H_{\mathrm{Nsig}_{V}}\right) \neq \emptyset\right)$ but no classical model $\left(\mathcal{C}\left(H_{G} \cup H_{\mathrm{Nsig}_{V}}\right)=\emptyset\right)$. It has been proven in [9] that $\mathrm{MCG}(G)$ is pseudotelepathic if and only if $G$ is not bipartite.

Example 3: In a complete graph $K_{n}$ of order $n$, there exists a non-empty set $D$ involved in a question $x \in\{0,1\}^{V}$ if and only if $|x|=1 \bmod 2$. With each such question $x$, the associated hyperedge is $e_{x}=\left\{(a \mid x) \in\{0,1\}^{V} \times\right.$ $\{0,1\}^{V}$ s.t. $\left.2|a| \neq|x|+1 \bmod 4\right\}$.

When $n=3$, the scenario obtained has vertices $(a \mid x)$, with $a, x \in\{0,1\}^{3}$.

- $H_{N s i i_{\{1,2,3\}}}={ }^{\min } \otimes_{i=1}^{3} H_{N s i g_{i}}$ where $H_{N s i g_{i}}=\{\{(0 \mid 0),(1 \mid 0)\},\{(0 \mid 1),(1 \mid 1)\}\}$ are the hyperedges ensuring non-signaling.

\footnotetext{
${ }^{3}$ The Foulis Randall product of scenarios [8] is the scenario $H_{A} \otimes H_{B}$ with vertices $V\left(H_{A} \otimes H_{B}\right)=V\left(H_{A}\right) \times V\left(H_{B}\right)$ and edges $E\left(H_{A} \otimes H_{B}\right)=E_{A \rightarrow B} \cup E_{A \leftarrow B}$ where $E_{A \rightarrow B}:=\left\{\cup_{a \in e_{A}}\{a\} \times f(a): e_{a} \in E_{A}, f: e_{A} \rightarrow E_{B}\right\}$ and $E_{A \leftarrow B}:=\left\{\cup_{b \in e_{A}} f(b) \times\{b\}:\right.$ $\left.e_{b} \in E_{b}, f: E_{B} \rightarrow E_{A}\right\}$. In the multipartite case there are several ways to define products, however they all correspond to the same non-locality constraints [8]. Therefore one can just consider the minimal product ${ }^{\min } \otimes_{i=1}^{n} H_{i}$ which has vertices in the cartesian product $V=\Pi V_{i}$ and edges $\cup_{k \in[1, n]} E_{k}$ where $E_{k}=\left\{\left(v_{1} \ldots, v_{n}\right), v_{i} \in e_{i} \forall i \neq k, v_{k} \in f(\vec{v})\right\}$ for some edge $e_{i} \in E\left(H_{i}\right)$ for every party $i \neq k$ and a function $\vec{v} \mapsto f(\vec{v})$ which assigns to every joint outcome $\vec{v}=\left(v_{1} \ldots v_{k-1}, v_{k+1}, \ldots v_{n}\right)$ an edge $f(\vec{v}) \in E\left(H_{k}\right)$ (the $k^{\text {th }}$ vertex is replaced by a function of the others).

${ }^{4}$ Note that for the questions $x$ for which there exists no $D$ involved in $x$, all the answers are allowed thus the constraints represented by the associated edge is a hyperedge of no-signaling scenario $H_{N s i g}$.
} 


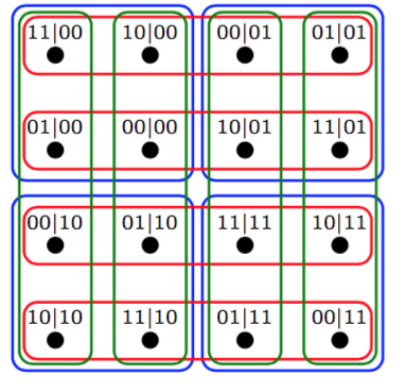

Figure 2: $H_{\mathrm{Nsig}_{2}}$ : hyperedges of the Bell scenario $B_{2,2,2}$ from [8]

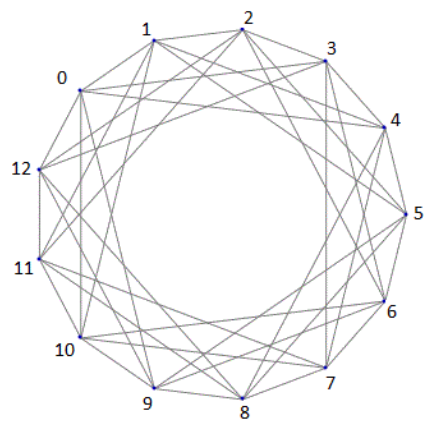

Figure 3: Paley Graph of order 13

- $H_{G}=\left\{e_{001}, e_{010}, e_{001}, e_{111}\right\}$ where

$$
\begin{aligned}
& e_{001}=\{(000 \mid 001),(011 \mid 001),(101 \mid 001),(110 \mid 001)\}, \\
& e_{010}=\{(000 \mid 010),(011 \mid 010),(101 \mid 010),(110 \mid 010)\}, \\
& e_{001}=\{(000 \mid 001),(011 \mid 001),(101 \mid 001),(110 \mid 001)\}, \\
& e_{111}=\{(100 \mid 111),(010 \mid 111),(001 \mid 111),(111 \mid 111)\} .
\end{aligned}
$$

Example 4: In the graph Paley 13 (see Figure 3), $\operatorname{Odd}(\{0,1,4\})=$ $\{2,7,8,9,11,12\}$ thus if $\{0,1,4\}$ is involved in $x$ i.e. $x_{i}=1$ for $i \in\{0,1,4\}$ and $x_{i}=0$ for $i \in\{2,7,8,9,11,12\}$ then the associated pseudo-telepathy game requires that the sum of the outputs of these nine players $\sum_{i \notin\{3,5,6,10\}} a_{i}$ has to be odd. This corresponds to 8 hyperedges $e_{j k l}$ for $j, k, l \in\{0,1\}$ in the contextuality scenario where $e_{j k l}=\left\{(a \mid x), \sum_{i \notin\{5,6,10\}} a_{i}=1 \bmod 2, x_{i}=1\right.$ for $i \in\{0,1,4\}, x_{i}=0$ for $\left.i \in\{2,7,8,9,11,12\}, x_{5}=j, x_{6}=k, x_{10}=l\right\}$.

For any contextuality scenario (hypergraph), one can choose an integer $n$, label the vertices with $(a \mid x)$ with $a, x \in\{0,1\}^{n}$, add some new vertices to have $2^{2 n}$ vertices, add the non signaling hyperedges induced by the labeling and try to find a model consistent with the original hyperedges and the nonsignaling ones. Thus it seems interesting to lift the properties observed in our model to the scenario itself, by considering the possible labelings. Note that as pseudotelepathy is a special case of nonlocality, for the families of scenarios that we build, the labelling and the non signaling condition are already fixed.

It is interesting to go back to the models of contextuality defined in [1] as the probabilistic contextuality is what was considered in [9] as it corresponds to investigating the possibility of simulating a probability distribution of a quantum strategy playing with graph states. 
Furthermore, the two other levels of contextuality can gain some new perspectives when viewed as games: indeed the possibilistic contextuality is similar to the fact that the players cannot give all the good answers with non zero probability using classical local strategies, and strong contextuality is similar to the case where classical players cannot win the game (even by giving a strict subset of the good answers).

Definition 3. An interpretation $p:\{0,1\}^{V} \times\{0,1\}^{V} \rightarrow[0,1]$ is $k$ multipartite if it can be obtained by a strategy without communication using nonlocal boxes that are at most $k$-partite: for any set $I \subset V$ with $|I| \leq k$, each player has access to one bit of a variable $\lambda_{I}\left(a_{I} \mid x_{I}\right)$ that has a no-signaling probability distribution.

In other words, a $k$-multipartite interpretation can be obtained with nosignaling correlations involving at most $k$ players. For example the strategy to win the Mermin game proposed in [17] where each pair among $n$ players share a (2-partite) non localbox and each player outputs the sum of his boxes' ouputs is a 2-multipartite interpretation. Similarly, the result in [11] where they prove that a probability distribution that can be obtained by 5 players measuring a quantum state cannot be simulated without communication using any number of bi-partite non local boxes shows that it is not a 2-multipartite interpretation. ${ }^{5}$

Definition 4 (multipartiteness width). A scenario has a multipartiteness width $k$ if it admits a $k$-multipartite interpretation but no $(k-1)$ multipartite interpretation.

In a contextual scenario, the more hyperedges one adds the less possible interpretations exist (the interpretations of a hypegraph with one extra hyperedge are a subset of the interpretations of the one with one less constraint). A scenario has a multipartiteness width $k$ if its hyperedges already forbids all the interpretations of a product of Bell scenarios on less than $k$ parties. For a scenario, having a classical interpretation means being decomposable : one can think of the probability distribution as local actors acting each on his bit and that's a classical interpretation. The multipartiteness width

\footnotetext{
${ }^{5}$ The probability distribution described in [11] corresponds to the quantum winning strategy on the graph state obtained from a cycle with 5 vertices.
} 
measure how non-decomposable a scenario is : it can not be decomposed with interpretations where each subspace has a small width.

It implies that the players cannot perfectly win the game if they have only quantum systems on less than $k$ qubits, this corresponds to using $k$ separable states as ressources as defined in [31].

Note that from the observations in [9] the multipartiteness width of the scenario generated by the Paley graph on 13 (see figure 3) is strictly larger than 4 .

In the next section, we will show how for the scenarios we describe, being able to give only good answers allows for simulation of the quantum distribution with random variables. Thus, the contextuality lies in the combinatorial structure of the graph and the three levels collapse for these games.

\section{Simulating a probability distribution is the same as winning the pseudo-telepathy graph game}

In [9] it was proven that for some graphs, the probability distributions of the quantum strategy using the graph states cannot be simulated using non local boxes on less than $k$ parties, we show here that any strategy that allows to win the game can be extended using random variables shared between neighbors (in the graph) to simulate the uniform probability distribution arising from the quantum strategy.

We start by describing a classical strategy CStrat based on shared random variables rather than quantum states. We show that CStrat is a winning strategy if and only if the graph is bi-partite. We also show that CStrat can be used to make any winning strategy a uniform winning strategy, i.e. each valid answer to a given question are equiprobable. We show that CStrat can be locally adapted to collaborative games on graphs that can be obtained by a sequence of local complementations.

Classical strategy (Cstrat): Given a graph $G=(V, E)$, pick uniformly at random $\lambda \in\{0,1\}^{V}$. Each player $u \in V$ receives a pair of bits $\left(\lambda_{u}, \mu_{u}\right)$, where $\mu_{u}=\sum_{v \in N_{G}(u)} \lambda_{u} \bmod 2$. Given a question $x \in\{0,1\}^{V}$, each player $u \in V$ locally computes and answers $a_{u}=\left(1-x_{u}\right) \cdot \lambda_{u}+x_{u} \cdot \mu_{u} \bmod 2$.

Lemma 5. Given a graph $G=(V, E)$ and a question $x \in\{0,1\}^{V}$, CStrat produces an answer uniformly at random in $\left\{a \in\{0,1\}^{V} \mid \exists D \subseteq S,(A \oplus\right.$ $\operatorname{Odd}(A \oplus D)) \cap S=\emptyset$ where $A=\operatorname{supp}(a)$ and $S=\operatorname{supp}(x)\}$. 
Proof: Given a graph $G=(V, E)$, a question $x \in\{0,1\}^{V}$ and $a \in\{0,1\}^{V}$, the probability that CStrat outputs $a$ is

$$
\begin{aligned}
p(a \mid x) & =p\left(\forall u \in V \backslash S, a_{u}=\lambda_{u}\right) p\left(\forall u \in S, a_{u}=\sum_{v \in N(u)} \lambda_{v} \bmod 2 \mid \forall u \in V \backslash S, a_{u}=\lambda_{u}\right) \\
& =p(A \backslash S=\Lambda \backslash S) p(A \cap S=\operatorname{Odd}(\Lambda) \cap S \mid A \backslash S=\Lambda \backslash S)
\end{aligned}
$$

where $S=\operatorname{supp}(x), A=\operatorname{supp}(a)$ and $\Lambda=\operatorname{supp}(\lambda)$. Since $p(A \backslash S=\Lambda \backslash S)=$ $\frac{1}{2^{n-|x|}}, p(a \mid x)=\frac{1}{2^{n-|x|}} p(A \cap S=\operatorname{Odd}(\Lambda \cap S \oplus \Lambda \backslash S) \cap S \mid A \backslash S=\Lambda \backslash S)$ $=\frac{1}{2^{n-|x|}} p(A \cap S=\operatorname{Odd}(D \oplus(A \backslash S)) \cap S \mid A \backslash S=\Lambda \backslash S)$, where $D=\Lambda \cap S$. If $A \cap S \neq \operatorname{Odd}(D \oplus(A \backslash S)) \cap S$ for all $D \subseteq S$, then $p(a \mid x)=0$. Otherwise, the set of subsets $D$ of $S$ which satisfy the condition is the affine space $\left\{D_{0} \oplus D \mid D \subseteq S \wedge \operatorname{Odd}(D) \cap S=\emptyset\right\}$, where $D_{0}$ is a fixed set which satisfies $A \cap S=\operatorname{Odd}\left(D_{0} \oplus A \backslash S\right) \cap S$. Thus the $p(a \mid x)=\frac{1}{2^{n-|x|}} \cdot \frac{|\{D \subseteq S \mid \operatorname{Odd}(D) \cap S=\emptyset\}|}{2^{|x|}}=$ $2^{|x|-r k_{G}(x)-n}$, which is independent of $a$, proving the uniformity of the answer. Finally notice $\exists D_{0} \subseteq S, A \cap S=\operatorname{Odd}\left(D_{0} \oplus(A \backslash S)\right) \cap S$ if and only if $\exists D_{1} \subseteq S,\left(A \oplus \operatorname{Odd}\left(A \oplus D_{1}\right)\right) \cap S=\emptyset$, by taking $D_{1}=D_{0} \oplus(A \cap S)$.

We consider some standard graph transformations: Given a graph $G=$ $(V, E)$ the local complementation [16] on a vertex $u \in V$ produces the graph $G * u=\left(V, E \oplus K_{N(v)}\right)$ where the sum is taken modulo 2 (it is the symmetric difference) and $K_{U}$ is the complete graph on $U \subset V$. G*u is obtained from $G$ by exchanging the edges by non edges and vice versa in the neighborhood of the vertex $u$. Pivoting using an edge $(u, v)$, is a sequence of three local complementations $G \wedge u v=G * u * v * u$. We denote by $\delta_{l o c}(G)[35,36,19]$ (resp. $\left.\delta_{\text {piv }}(G)\right)$ the minimum degree taken over all graphs that can be obtained from $G$ through some sequence of local complementations (edge pivots).

Given the shared randomness $\left(\lambda_{v}, \mu_{v}\right)_{v \in V}$ associated with $G$, if player $u$ replaces its first bit by the XOR of its two bits, and each of his neighbors replaces his second bit by the XOR of his two bits, one gets the shared randomness associated with $G * u$. (proof given in Appendix)

Lemma 6. Given the probability distribution $\left(\lambda_{v}, \mu_{v}\right)_{v \in V}$ associated with $G$, if player $u$ replaces its first bit by the XOR of its two bits, and each of its neighbors replaces their second bit by the XOR of their two bits, one gets the probability distribution associated with $G * u$.

Thus the probability distribution corresponding to the classical strategy for $G$ can be locally transformed into the probability distribution associated with the $G * u$, thus one can use local complementation to optimise the cost of 
preparing the shared randomness. For instance the classical strategy CStrat for a graph $G$ requires shared random bits on at most $\Delta_{l o c}(G)+1$ players, where $\Delta_{l o c}(G)=\min \left(\Delta\left(G^{\prime}\right)\right.$, s.t. $\left.\exists u_{1}, \ldots, u_{k}, G^{\prime}=G * u_{1} * \ldots * u_{k}\right)$ and $\Delta(G)$ is its maximum degree. If there is no pre-shared random bits, the probability distribution can be prepared using at most $2|G|_{l o c}$ communications in-between the players, where $|G|_{l o c}=\min \left(\left|G^{\prime}\right|\right.$, s.t. $\left.\exists u_{1}, \ldots, u_{k}, G^{\prime}=G * u_{1} * \ldots * u_{k}\right)$ is the minimum number of edges by local complementation.

Now we show how, using the classical strategy CStrat, one can simulate the quantum strategy QStrat given an oracle that provides only good answers.

Lemma 7. For any collaborative game on a graph $G$, for any strategy $Q$ that never loses, there exists a strategy $Q^{\prime}$ using the outputs of $Q$ and shared random variables that simulate $\boldsymbol{Q S t r a t}$.

\section{Proof:}

Given a collaborative graph game on a graph $G$, let $Q$ be a strategy that always outputs permissible outputs for any set of inputs $x$, so we have pairs $(a \mid x) \notin \mathcal{L}$. We consider the strategy which combines $Q$ and CStrat for this graph: For a given question $x, Q^{\prime}$ outputs the XOR of the $Q$ answer and CStrat answer for $x$. First we prove that such an answer is a valid answer and then the uniform probability among the possible answer to a given question. Given a question $x \in\{0,1\}^{V}$, suppose $Q^{\prime}$ outputs $a^{\prime} \in\{0,1\}^{V}: \forall u \in V$, $a_{u}^{\prime}=a_{u}+\left(1-x_{u}\right) \lambda_{u}+x_{u} \mu_{u}$ where $a_{u}$ is the answer produced by $Q$ and $\lambda$ and $\mu$ are as defined in the classical strategy. By contradiction, assume $\left(a^{\prime} \mid x\right) \in \mathcal{L}$, so there exists $D$ involved in $x$ such that $\sum_{u \in \operatorname{loc}(D)} a_{u}^{\prime}=|G[D]|+1 \bmod 2$.

$\sum_{u \in \operatorname{loc}(D)} a_{u}^{\prime}=\sum_{u \in \operatorname{loc}(D)}\left(a_{u}+\left(1-x_{u}\right) \lambda_{u}+x_{u} \mu_{u}\right) \bmod 2=$
$\sum_{u \in \operatorname{loc}(D)} a_{u}+\sum_{u \in \operatorname{loc}(D) \backslash \operatorname{supp}(x)} \lambda_{u}+\sum_{u \in \operatorname{loc}(D) \cap \operatorname{supp}(x)} \mu_{u} \bmod 2$
$\sum_{u \in \operatorname{loc}(D)} a_{u}+\sum_{u \in \operatorname{Odd}(D)} \lambda_{u}+\sum_{u \in D} \sum_{v \in N(u)} \lambda_{v} \bmod 2=\sum_{u \in \operatorname{loc}(D)} a_{u}+$
$\sum_{u \in \operatorname{Odd}(D)} \lambda_{u}+\sum_{v \in \operatorname{Odd}(D)} \lambda_{v} \bmod 2=\sum_{u \in \operatorname{loc}(D)} a_{u} \bmod 2$.
$(a \mid x) \in \mathcal{L}$ which is a contradiction thus $p\left(a^{\prime} \mid x\right)=0$ if $\left(a^{\prime} \mid x\right) \in \mathcal{L}$. Now we prove that $p\left(a^{\prime} \mid x\right)=2^{|x|-n-r k_{G}(x)}$. First assume $Q$ is determinist, thus $p\left(a^{\prime} \mid x\right)$ is the probability that the classical strategy outputs $a+a^{\prime}:=\left(a_{u}+a_{u}^{\prime} \bmod 2\right)_{u \in V}$. Since this probability is non zero it must be $2^{|x|-n-r k_{G}(x)}$. If $Q$ is probabilistic, $p\left(a^{\prime} \mid x\right)=$ $\sum_{a \in\{0,1\}^{V}} p(Q$ outputs $a$ on $x) p\left(\right.$ classical strategy outputs $a+a^{\prime}$ on $\left.x\right) \leq$ $2^{|x|-n-r k_{G}(x)} \sum_{a \in\{0,1\}^{v}} p(Q$ outputs $a$ on $x) \leq 2^{|x|-n-r k_{G}(x)}$. Thus each answer $a$ produced by the strategy on a given question $x$ is s.t. $(a \mid x) \notin \mathcal{L}$ and occurs with probability at most $2^{|x|-n-r k_{G}(x)}$. Since 
$\left|\left\{a \in\{0,1\}^{V} \mid(a \mid x) \notin \mathcal{L}\right\}\right|=2^{|x|-n-r k_{G}(x)}$, each of the possible answers is produced by the strategy and occurs with probability $2^{|x|-n-r k_{G}(x)}$.

\section{Locally equivalent games}

A pseudo telepathy game $\mathcal{G}$ locally simulates another pseudo telepathy game $\mathcal{G}^{\prime}$ if any winning strategy for $\mathcal{G}$ can be locally turned into a winning strategy for $\mathcal{G}^{\prime}$ :

Definition 8 (Local Simulation). Given two pseudo telepathy games $\mathcal{G}$ and $\mathcal{G}^{\prime}$ on a set $V$ of players which sets of losing pairs are respectively $\mathcal{L}_{\mathcal{G}}$ and $\mathcal{L}_{\mathcal{G}^{\prime}}, \mathcal{G}$ locally simulates $\mathcal{G}^{\prime}$ if for all $u \in V$, there exist $f_{1}, \ldots, f_{n}$ : $\{0,1\} \rightarrow\{0,1\}$ and $g_{1}, \ldots, g_{n}:\{0,1\} \times\{0,1\} \rightarrow\{0,1\}$ s.t. $\forall x, a \in\{0,1\}^{V}$ $(g(a, x), x) \in \mathcal{L}_{\mathcal{G}^{\prime}} \Rightarrow(a \mid f(x)) \in \mathcal{L}_{\mathcal{G}}$ where $f(x)=\left(f_{u}\left(x_{u}\right)\right)_{u \in V}$ and $g(a, x)=$ $\left(g_{u}\left(a_{u}, x_{u}\right)\right)_{u \in V}$.

Assuming $\mathcal{G}$ locally simulates $\mathcal{G}^{\prime}$ and that the players have a strategy to win $\mathcal{G}$, the strategy for $\mathcal{G}^{\prime}$ is as follows: given an input $x$ of $\mathcal{G}^{\prime}$, each player $u$ applies the preprocessing $f_{u}$ turning her input $x_{u}$ into $f_{u}\left(x_{u}\right)$, then they collectively play the game $\mathcal{G}$ with this input $f(x)$ getting an output $a$ s.t. $(a \mid f(x)) \notin \mathcal{L}_{\mathcal{G}}$. Finally each player $u$ applies a postprocessing $g_{u}$ which depends on her output $a_{u}$ and her initial input $x_{u}$ to produce the output $g_{u}\left(a_{u}, x_{u}\right)$ to the game $\mathcal{G}^{\prime}$. This output is valid since, by contradiction, $(g(a, x), x) \in \mathcal{L}_{\mathcal{G}^{\prime}}$ would imply $(a \mid f(x)) \in \mathcal{L}_{\mathcal{G}}$.

Definition 9 (Local Equivalence). $\mathcal{G}$ and $\mathcal{G}^{\prime}$ are locally equivalent games if $\mathcal{G}$ locally simulates $\mathcal{G}^{\prime}$ and $\mathcal{G}^{\prime}$ locally simulates $\mathcal{G}$.

In the following we give two examples of locally equivalent games (the proofs of equivalence are given in Appendix): first we show that the games associated with the complete graphs are locally equivalent to Mermin parity games, and then that pivoting, a graph theoretical transformation, produces a graph game locally equivalent to the original one:

Lemma 10. For any $n$, the game associated with the complete graph $K_{n}$ is locally equivalent to the Mermin parity game on $n$ players.

Lemma 11. Given a graph $G=(V, E)$ and $(u, v) \in E$, the games associated with $G$ and $G \wedge$ uv are locally equivalent.

Therefore, the important quantity for the pre-shared randomness for the strategies defined with a graph is $\Delta_{\text {piv }}(G)=$ $\min \left\{\Delta\left(G^{\prime}\right), G^{\prime}\right.$ pivot equivalent to $\left.G\right\}$. 


\section{Scenarios with linear multipartiteness width}

We prove that there exist contextuality scenarios with linear multipartiteness width. We use a graph property called $k$-odd domination which is related [9] to the classical simulation of the quantum probability distribution obtained by playing the associated graph game. Since bipartite graphs correspond to graph games that can be won classically [9], we focus on the non-bipartite case by showing that there exist non-bipartite $0.11 n$-odd dominated graphs of order $n$.

Definition 12 (k-odd domination [9]). A graph $G=(V, E)$ is $k$-odd dominated (k-o.d.) iff for any $S \in\left(\begin{array}{l}V \\ k\end{array}\right)$, there exists a labelling of the vertices in $S=\left\{v_{1}, \ldots, v_{k}\right\}$ and $C_{1}, \ldots C_{k}$, s.t. $\forall i, C_{i} \subseteq V \backslash S$ and $\operatorname{Odd}\left(C_{i}\right) \cap\left\{v_{i}, \ldots v_{k}\right\}=\left\{v_{i}\right\}$ and $C_{i} \subseteq \operatorname{Even}\left(C_{i}\right)$.

Lemma 13. For any $k \geq 0, r \geq 0$ and any graph $G=(V, E)$ a graph of order $n$ having two distinct independent sets $V_{0}$ and $V_{1}$ of order $\left|V_{0}\right|=\left|V_{1}\right|=\left\lfloor\frac{n-r}{2}\right\rfloor$, $G$ is $k$-odd dominated if for any $i \in\{0,1\}$, and any non-empty $D \subseteq V \backslash V_{i}$, $\left|\operatorname{Odd}_{G}(D) \cap V_{i}\right|>k-|D|$

Proof: Given $S_{0} \subseteq V_{0}, S_{1} \subseteq V_{1}$, and $S_{2} \subseteq V_{2}=V \backslash\left(V_{0} \cup V_{1}\right)$ s.t. $\left|S_{0}\right|+$ $\left|S_{1}\right|+\left|S_{2}\right|=k$, we show that for any $u \in S=S_{0} \cup S_{1} \cup S_{2}$, there exists $C_{u} \subseteq V \backslash S$ s.t. $\operatorname{Odd}\left(C_{u}\right) \cap S=\{u\}$ and $C_{u} \subseteq \operatorname{Even}\left(C_{u}\right)$. For any $u \in S$, there exists $i \in\{0,1\}$ s.t. $u \in S_{i} \cup S_{2}$. Let $L_{i}: 2^{S_{i} \cup S_{2}} \rightarrow 2^{V_{1-i} \backslash S_{1-i}}$ be the function which maps $D \subseteq S_{i} \cup S_{2}$ to $L_{i}(D)=O d d_{G}(D) \cap\left(V_{1-i} \backslash S_{1-i}\right)$. $L_{i}$ is linear according to the symmetric difference. $L_{i}$ is injective: for any $D \subseteq S_{i} \cup S_{2}, \operatorname{Odd}(D) \cap\left(V_{1-i} \backslash S_{1-i}\right)=\emptyset$ implies $\operatorname{Odd}(D) \cap V_{1-i} \subseteq S_{1-i}$, thus $\left|\operatorname{Odd}(D) \cap V_{1-i}\right| \leq\left|S_{1-i}\right|$. notice that $|D| \leq\left|S_{i}\right|+\left|S_{2}\right|$, so $\left|\operatorname{Odd}(D) \cap V_{1-i}\right| \leq$ $\left|S_{1-i}\right| \leq\left|S_{0}\right|+\left|S_{1}\right|+\left|S_{2}\right|-|D|=k-|D|$, so $D=\emptyset$. The matrix representing $L_{i}$ is nothing but the submatrix $\Gamma_{\left[S_{i} \cup S_{2}, V_{1-i} \backslash S_{1-i}\right]}$ of the adjacency matrix $\Gamma$ of $G$. So its transpose $\Gamma_{\left[V_{1-i} \backslash S_{1-i}, S_{i} \cup S_{2}\right]}$ is surjective which means that the corresponding linear map $L_{i}^{T}: 2^{V_{1-i} \backslash S_{1-i}} \rightarrow 2^{S_{i} \cup S_{2}}=C \mapsto \operatorname{Odd}_{G}(C) \cap\left(V_{1-i} \backslash\right.$ $\left.S_{1-i}\right)$ is surjective, so $\exists C_{u} \subseteq V_{1-i} \backslash S_{1-i}$ s.t. $\operatorname{Odd}_{G}\left(C_{u}\right) \cap\left(S_{i} \cup S_{2}\right)=\{u\}$, which implies, since $V_{1-i}$ is an independent set, that $\operatorname{Odd}_{G}\left(C_{u}\right) \cap S=\{u\}$ and $C_{u} \subseteq \operatorname{Even}\left(C_{u}\right)$.

Theorem 14. For any even $n>n_{0}$, there exists a non-bipartite $\lfloor 0.110 n\rfloor$-odd dominated graph of order $n$. 
Proof: Given $n, r \leq n$ s.t. $r=n \bmod 2$, and $k \geq 0$. Let $p=(n-r) / 2$, and let $G=\left(V_{0} \cup V_{1} \cup V_{2}, E\right)$ s.t. $\left|V_{0}\right|=\left|V_{1}\right|=p,\left|V_{2}\right|=r$ be a random graph on $n$ vertices s.t. for any $u \in V_{i}, v \in V_{j}$ there is an edge between $u$ and $v$ with probability 0 if $i=j$ and with probability $1 / 2$ otherwise. For any $i \in\{0,1\}$, and any non empty $D \subseteq V \backslash V_{i}$ s.t. $|D| \leq k$, let $A_{D}^{(i)}$ be the bad event $\left|\operatorname{Odd}_{G}(D) \cap V_{i}\right| \leq k-|D|$. Since each vertex of $V_{i}$ is in $\operatorname{Odd}_{G}(D)$ with probability $1 / 2, \operatorname{Pr}\left(A_{D}^{(i)}\right)=\sum_{j=0}^{k-|D|}\left(\begin{array}{l}p \\ j\end{array}\right) 2^{-p} \leq 2^{p\left[H\left(\frac{k-|D|}{p}\right)-1\right]}$. Another bad event is that $G$ is bipartite which occurs with probability less than $\left(\frac{7}{8}\right)^{p r}$. Indeed, the probability that given $u \in V_{0}, v \in V_{1}, w \in V_{2},(u, v, w)$ do not form a triangle is $\frac{7}{8}$, so given a bijection $f: V_{0} \rightarrow V_{1}$, the probability that $\forall u \in V_{0}, \forall w \in V_{2},(u, f(u), w)$ do not form a triangle is $\left(\frac{7}{8}\right)^{p r}$. Let $X$ be the number of bad events. $E[X]=2 \sum_{d=1}^{k}\left(\begin{array}{c}p+r \\ d\end{array}\right) \sum_{j=0}^{k-d}\left(\begin{array}{c}p \\ j\end{array}\right) 2^{-p}+\left(\frac{7}{8}\right)^{p r} \leq$ $2 \sum_{d=1}^{k} 2^{(p+r) H\left(\frac{d}{p+r}\right)+p H\left(\frac{k-d}{p}\right)-p}+\left(\frac{7}{8}\right)^{p r} \leq 2 \sum_{d=1}^{k} 2^{p H\left(\frac{d}{p+r}\right)+p H\left(\frac{k-d}{p}\right)-p+r}+\left(\frac{7}{8}\right)^{p r}$. The function $d \mapsto p H\left(\frac{d}{p+r}\right)+p H\left(\frac{k-d}{p}\right)-p+r$ is maximal for $d=\frac{k(p+r)}{2 p+r}$. Thus, $E[X] \leq 2 k 2^{2 p H\left(\frac{k}{2 p+r}\right)-p+r}+\left(\frac{7}{8}\right)^{p r}$. By taking $r=1$, and $k=0.11 n=$ $0.11(2 p+1), E[X]<1$ when $p$ large enough, thus $G$ has no bad event with a non zero probability.

Corollary 15. There exist contextuality scenarios with linear multipartiteness width: for any even $n>n_{0}$, there exist graph games on $n$ players producing contextuality scenarios of multipartiteness width at least $\lfloor 0.11 n\rfloor$.

Proof: Using the result from [9], for any non bipartite graph of order $n$ being $0.11 n$-o.d ensures that the probability distribution obtained by using the quantum strategy cannot be simulated using non local boxes involving at most $0.11 n$ parties. Thus lemma 7 allows to conclude that the associated pseudo-telepathy game cannot be won classically. Therefore there is no interpretation that is $k$-multipartite with $k<0.11 n$ which means that the contextuality scenario has linear width.

\section{Conclusion}

We have shown that there exist graphs with linear multipartiteness width, however the proof is non constructive and the best known bound for explicit families is logarithmic. A natural future direction of research would be to find explicit families with linear multipartiteness width or to improve the bounds proven for the Paley graph states. An other important question is to consider 
lower bounds for the scenarios associated with the graph games. A promising area of investigation for multipartite scenarios is: what happens if we limit the width of shared randomness? Indeed, for the proof of how winning the game allows to simulate the quantum probability distributions, one needs only shared random variables that are correlated in local neighborhoods in the graph. One can also consider the link with building entanglement witnesses for graph states, generalizing the construction of [33]. It would be also very interesting to link the multipartiteness width with the structures of the groups of the associated binary linear system defining the two-player bipartite non-local games [47]. Very recently, Chao and Reichardt [20] proposed a test separating quantum theory form $k$-local theories, where players can use $k$-local boxes. A future work could consist in defining new tests associated with our games, proving bounds on how far they are from being winnable in $k$-local theories. Finally, one can expect that the multipartiteness width of the Paley graph states might have cryptographic applications to ensure security against cheating for some protocols for example.

\section{Acknowledgements.}

We would like to thank an anonymous reviewer for noticing a mistake in an earlier version and helpful comments. This research was supported in part by the ANR-14-CE25-0006 project of the French National Research Agency and by PEPS INS2I 2016 JCQ.

[1] S. Abramsky and A. Brandenburger. The sheaf theoretic structure of non locality and contextuality. New Journal of Physics 13 113036, 2011.

[2] S. Abramsky, R.S. Barbosa, G. Carù, and S. Perdrix. A complete characterization of all-versus-nothing arguments for stabilizer states, Phil. Trans. R. Soc. A, 375(2106), 20160385, 2017.

[3] S Abramsky, RS Barbosa, G Carù, N de Silva, K Kishida and S Mansfield Minimum quantum resources for strong non-locality. Proc. TQC 2017, Leibniz International Proceedings in Informatics (LIPIcs), 9:1-9:20, 2017.

[4] S. Abramsky, R.S. Barbosa and S. Mansfield. Quantifying contextuality via linear programming, Informal Proceedings of Quantum Physics \& Logic, 2016. 
[5] S. Abramsky, R.S. Barbosa and S. Mansfield. The contextual fraction as a measure of contextuality, Phys. Rev. Lett. 119, 050504 (2017)

[6] S Abramsky, L Hardy Logical bell inequalities. Phys. Rev. A 85, 062114, 2012.

[7] S. Abramsky, S. Mansfield, R.S. Barbosa. The cohomology of non-locality and contextuality. Electronic Proceedings in Theoretical Computer Science, 95, 1-14, 2012.

[8] A. Acín, T. Fritz, A. Leverrier, and A. Belén Sainz. A combinatorial approach to nonlocality and contextuality. Comm. Math. Phys. 334(2), 533-628, 2015.

[9] A. Anshu and M. Mhalla. Pseudo-telepathy games and genuine NS k-way nonlocality using graph states Quantum Information and Computation, Vol. 13, No. 9, 10 0833-0845 Rinton Press, 2013.

[10] A. Badanidiyuru, J. Langford, A. Slivkins. Resourceful Contextual Bandits. http://arxiv.org/abs/1402.6779, COLT, 2014.

[11] J. Barrett and S. Pironio. Popescu-Rohrlich correlations as a unit of nonlocality. Phys. Rev. Lett. 95, 140401, 2005.

[12] J.S. Bell. On the Einstein-Podolsky-Rosen paradox. Physics 1, 195200,1964 .

[13] G. Brassard, A. Broadbent and A. Tapp. Multi-Party Pseudo-Telepathy 8th International Workshop, WADS 2003, Ottawa, Ontario, Canada, July 30 - August 1, Proceedings, 2003.

[14] J. Bermejo-Vega, N. Delfosse, Dan E. Browne, C. Okay, and R. Raussendorf. Contextuality as a resource for models of quantum computation with qubits. Phys. Rev. Lett. 119, 120505, 2017.

[15] Anne Broadbent, Joseph Fitzsimons, and Elham Kashefi. Universal blind quantum computation. In 50th Annual IEEE Symposium on Foundations of Computer Science, FOCS 2009, 2009.

[16] A. Bouchet. Connectivity of isotropic systems. In New York Academy of Sciences, editor, Proceedings of the third international conference on Combinatorial mathematics, pages 81-93,1989. 
[17] A. Broadbent and A. A. Methot. On the power of non-local boxes Theoretical Computer Science C 358: 3-14, 2006.

[18] D. E. Browne, E. Kashefi, M. Mhalla, and S. Perdrix. Generalized flow and determinism in measurement-based quantum computation. New Journal of Physics (NJP), 9(8), 2007. URL: http://iopscience.iop.org/1367-2630/9/8/250/fulltext/.

[19] D. Cattanéo, S. Perdrix. Minimum Degree up to Local Complementation: Bounds, Parameterized Complexity, and Exact Algorithms. In International Symposium on Algorithms and Computation (pp. 259-270). Springer Berlin Heidelberg. 2015.

[20] R. Chao and B. W. Reichardt Test to separate quantum theory from non-signaling theories arXiv: https://arxiv.org/abs/1706.02008, 2017.

[21] B. Coecke. From quantum foundations via natural language meaning to a theory of everything. arXiv:1602.07618v1, 2016.

[22] V. Danos, E. Kashefi, P. Panangaden, and S.Perdrix. Extended Measurement Calculus. Cambridge University Press, 2010.

[23] V. Danos and E. Kashefi. Determinism in the one-way model. Physical Review A, 74(052310), 2006.

[24] E. Dzhafarov, S. Jordan , R. Zhang, V. Cervante. Contextuality from Quantum Physics to Psychology. Advanced Series on Mathematical Psychology: Volume 6, 2016.

[25] D. J. Foulis and C. H. Randall. Empirical logic and tensor products. J. Mathematical Phys. 5 , 9:20. MR683888., 1981.

[26] T. Fritz, A. B. Sainz, R. Augusiak, J. Bohr Brask, R. Chaves, A. Leverrier, and A. Acín. Local orthogonality as a multipartite principle for quantum correlations Nature Communications 4, 2263 , 2013.

[27] S. Gravier, J. Javelle, M. Mhalla, and S. Perdrix. Quantum secret sharing with graph states. In Antonín Kucera, Thomas A. Henzinger, Jaroslav Nesetril, Tomás Vojnar, and David Antos, editors, MEMICS, volume 7721 of Lecture Notes in Computer Science, pages 15-31. Springer, 2012. 
[28] A. Grudka, K. Horodecki, M. Horodecki, P. Horodecki, R. Horodecki, P. Joshi, W. Klobus, A. Wójcik. Quantifying contextuality Phys. Rev. Lett. 112, 120401, 2014.

[29] D. M. Greenberger, M. A. Horne, A. Shimony, and A. Zeilinger. Bell's theorem without inequalities. American Journal of Physics 58, 1131, 1990.

[30] P. Gnaciński, M. Rosicka, R. Ramanathan, K. Horodecki, M. Horodecki, P. Horodecki, S. Severini. Linear game non-contextuality and Bell inequalities - a graph-theoretic approach. e-print arXiv:1511.05415 Nov, 2015.

[31] O. Gühne, G.Tóth, H.J. Briegel. Multipartite entanglement in spin chains. New Journal of Physics, 7(1), 229. ,2005.

[32] L. Hardy. Nonlocality for two particles without inequalities for almost all entangled states. Physical Review Letters 71 , 1665:1668, 1993.

[33] M. Hein,W. Dür, J. Eisert, R. Raussendorf, M. Nest and H. J. Briegel. Entanglement in graph states and its applications. arXiv preprint quantph/0602096, 2006.

[34] M. Howard, J.J. Wallman, V. Veitch and J. Emerson. Contextuality supplies the "magic" for quantum computation. Nature 510, 351-355, 2014.

[35] P. Høyer, M. Mhalla, and S. Perdrix. Resources required for preparing graph states. In Proceedings of ISAAC06, LNCS, volume 4288, pages 638-649, 2006.

[36] J. Javelle, M. Mhalla, and S. Perdrix. On the minimum degree up to local complementation: Bounds and complexity. In MartinCharles Golumbic, Michal Stern, Avivit Levy, and Gila Morgenstern, editors, Graph-Theoretic Concepts in Computer Science (WG), volume 7551 of Lecture Notes in Computer Science, pages 138-147. Springer Berlin Heidelberg, 2012.

[37] Y.-C.Liang, D. Rosset, J-D. Bancal, G.Pütz, T.J. Barnea and N. Gisin. Family of Bell-like inequalities as device-independent witnesses for entanglement depth. Physical review letters, 114(19), 190401, 2015. 
[38] M. McKague. Interactive proofs for bqp via self-tested graph states. Theory of Computing, 12(3):1-42, 2016.

[39] A. Marin, D. Markham, S. Perdrix. Access Structure in Graphs in High Dimension and Application to Secret Sharing. In 8th Conference on the Theory of Quantum Computation, Communication and Cryptography (p. 308), 2013.

[40] D. Markham and B. C. Sanders. Graph states for quantum secret sharing. Physical Review A, 78:042309, 2008.

[41] N. D. Mermin. Extreme quantum entanglement in a superposition of macroscopically distinct states. Physical Review Letters, 65(15):18381849, 1990.

[42] M. Mhalla, M. Murao, S. Perdrix, M. Someya, and P. Turner. Which graph states are useful for quantum information processing? In Theory of Quantum Computation, Communication and Cryptography. (TQC'11), Lecture Notes in Computer Science, 2011.

[43] M. Mhalla and S. Perdrix. Graph States, Pivot Minor, and universality of (X-Z) Measurements. IJUC 9 (1-2) : 153-171, 2013.

[44] S. Perdrix, L. Sanselme. Determinism and Computational Power of Real Measurement-based Quantum Computation 21st International Symposium on Fundamentals of Computation Theory (FCT'17). 2017.

[45] R. Raussendorf Contextuality in measurement-based quantum computation. PhysRevA . 88, 022322, 2013.

[46] R. Raussendorf and H. J. Briegel. A one-way quantum computer. Phys. Rev. Lett., 86:5188-5191, 2001.

[47] W. Slofstra. Tsirelson's problem and an embedding theorem for groups arising from non-local games e-print arXiv:1606.03140, 2106.

[48] R. W. Spekkens, D. H. Buzacott, A. J. Keehn, B. Toner, and G. J. Pryde. Contextuality Powers Parity-Oblivious Multiplexing. Phys. Rev. Lett. 102, 010401, 2009. 
[49] W. Zeng and P. Zahg, Contextuality and the weak axiom of the theory of choice. Quantum Interactions proc. Volume 9535 of the series Lecture Notes in Computer Science pp 24-35, 2016. 